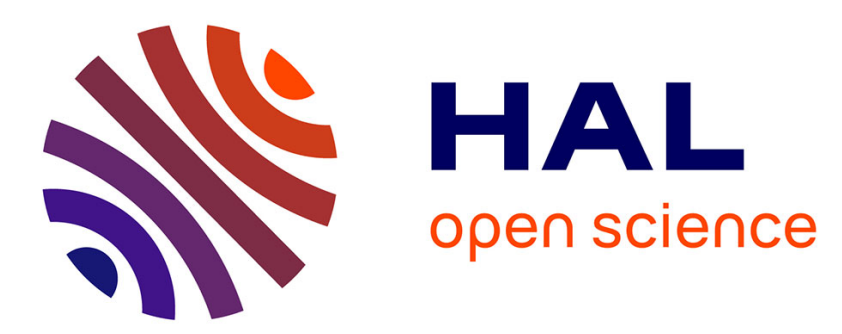

\title{
A quasi steady state method for solving transient Darcy flow in complex 3D fractured networks accounting for matrix to fracture flow
}

B Noetinger

\section{- To cite this version:}

B Noetinger. A quasi steady state method for solving transient Darcy flow in complex 3D fractured networks accounting for matrix to fracture flow. Journal of Computational Physics, 2015, 283, pp.205 - 223. 10.1016/j.jcp.2014.11.038 . hal-01115492

\author{
HAL Id: hal-01115492 \\ https://hal.science/hal-01115492
}

Submitted on 11 Feb 2015

HAL is a multi-disciplinary open access archive for the deposit and dissemination of scientific research documents, whether they are published or not. The documents may come from teaching and research institutions in France or abroad, or from public or private research centers.
L'archive ouverte pluridisciplinaire HAL, est destinée au dépôt et à la diffusion de documents scientifiques de niveau recherche, publiés ou non, émanant des établissements d'enseignement et de recherche français ou étrangers, des laboratoires publics ou privés. 


\title{
A quasi steady state method for solving transient Darcy flow in complex $3 D$ fractured
} networks accounting for matrix to fracture flow

\author{
B. Nœtinger* \\ IFP Energies nouvelles \\ 169 4, avenue de Bois-Préau \\ 92852 Rueil-Malmaison Cedex - France
}

(Dated: August 8, 2014)

Modeling natural Discrete Fracture Networks (DFN) receives more and more attention in applied geosciences, from oil and gas industry, geothermal recovery. The fractures may be either natural, or artificial in case of well stimulation. Accounting for the flow inside the fracture network, and accounting for the transfers between the matrix and the fractures, with the same level of accuracy is an important issue for calibrating the wells architecture and for setting up optimal resources recovery strategies. Recently, we proposed an original method allowing to model transient pressure diffusion in the fracture network only. The matrix was assumed to be impervious. A systematic approximation scheme was built, allowing to model the initial DFN by a set of $N$ unknowns located at the intersection between fractures. The higher $N$, the higher the accuracy of the model. The lowest order approximation $N=1$ appears under the form of solving a transient problem in a resistor/capacitor network, a so-called pipe network. Its topology is the same as the network of geometrical intersections between fractures.

In this paper, we generalize this approach in order to account for fluxes from matrix to fractures. We show that in the case of well separated time scales between matrix and fractures, the preceding model need only to be slightly modified in order to incorporate these fluxes. The additional knowledge of the so called matrix to fracture transfer function allows to modify the mass matrix that becomes a time convolution operator. This is reminiscent of existing space averaged transient dual porosity models.

Keywords: Flow in Fractured media, Discrete fracture network, Low permeability matrix, Quasi steady state, Dual porosity, Transfer function, Laplace transform

*Electronic address: benoit.noetinger@ifpen.fr 


\section{INTRODUCTION}

Modeling of fluid flows including heat or chemical transfers into naturally fractured rocks using explicit descriptions of fractured media (DFN) is becoming increasingly popular among geoscientists. This growing interest is due to a wide range of applications in various industries, to a better characterization of fracture networks, and evidently to the increasing computing power. In the common practice, these detailed descriptions are used to build and to calibrate a so called "double porosity model" that is designed to manage field applications. This class of double porosity models corresponds to the large scale homogenized version of the Darcy equations in the fractured medium, coupled to a linear transfer model with the matrix that is acting as a reservoir. These models, which were proposed in the early 60 's by Barenblatt et al [2] remain still the base of most industrial fluid flow simulators [3-10]. Homogenization techniques [1114], or Volume averaging techniques [15-17] allow a formal derivation of the double porosity equations, starting from the detailed DFN, at least in the Darcy hypothesis, and in the case of a well connected fracture network. Numerical solution of the associated closure problems permits to evaluate the parameters of the dual porosity model as a function of the geometry of the DFN. Useful connections with random walk theory providing efficient computational tools were made by several authors [18-22]. In the case of badly connected networks, modelling approaches involving percolation theory background are more appropriate [27-29]. But a complete workflow remains to be developed, especially if strong couplings with the matrix are involved, and in situations in which non linear transfers, like multiphase flow, are to be accounted for [8-10]. Direct simulations of flows in 2D or 3D DFN were already performed by several groups $([5,30-40])$. The underlying numerical methods involve finite volume, finite elements techniques. Some groups intend to couple the high resolution DFN model with a flow in the matrix [41].

Here, we focus on the simplest problem: fractures (here $2 D$ objects like closed polygons or ellipses of small thickness $\varepsilon$ of high typical conductivity $C_{f}=k_{f} \times \varepsilon$ are embedded in a $3 D$ matrix having a low permeability $k_{m} \ll k_{f}$ that will be supposed as being uniform for sake of simplicity. The fractures are supposed to be well connected (FIG. 1). Our goal is to solve linear diffusion equation within such a medium. Considering large cases involving thousands of intersecting fractures, the main difficulty of direct numerical solution techniques is to get an automated meshing fulfilling the quality requirements of the associated discretization scheme $[5,40]$. Even if this practical question is solved, the overall number of degrees of freedom remain equal to the number of fractures, say $N$, times the typical number of cells $N_{\text {typ }}$ used to mesh every fracture (typically $N_{t y p} \simeq$ several hundred). The number of associated matrix elements should scale as $N \times N_{t y p}^{3 / 2}$. Getting a numerical solution of a 10 millions fractures problem will imply thus solving close to several billion equations. This justifies developing approximation methods in which the number of degrees of 
freedom remains close to the total number of connected intersections $N_{\cap}$. This was done in [1] assuming an impervious matrix with $k_{m}=0$. In that paper, it was shown that a systematic approximation scheme can be built that involves $N_{p}$ unknowns that describe the trace of pressure at the intersections between fractures with increasing accuracy. At first order, $N_{p}=1$, which is equivalent to suppose that the pressure profiles at the intersection are uniform, the so called pipe network model is recovered. The resulting equations posses the structure of a resistor/capacitor network involving the total number of connected intersections $N_{\cap}$. The main physical assumption is that the considered time scales are much more greater that a typical diffusion time over one single fracture. The resulting set of equations reads:

$$
\forall i=1, N_{\cap}, m=1, \infty, \quad \sum_{j \in J(i)} \sum_{n=1}^{\infty} K_{i j}^{m n} \times \frac{d P_{j}^{n}(t)}{d t}=\sum_{j \in J(i)} \sum_{n=1}^{\infty} T_{i j}^{m n} \times P_{j}^{n} .
$$

Here, the integers $\mathrm{i}$ and $\mathrm{j}$ label the intersections, $J(i)$ is the ensemble of the labels of intersections directly connected to the i-th one. So as any intersection involves two different fractures, the set $J(i)$ involves the whole set of intersections belonging to both fractures intersecting at $\mathrm{i}$. Labels $\mathrm{m}$ and $\mathrm{n}$ indicate the degrees of freedom corresponding to the order of approximation of the pressure profiles at intersections. $N_{\cap i}$ corresponds to all the other intersections than $\mathrm{i}$ belonging to the pair of fractures the intersection of which is the $\mathrm{i}$ th intersection. The mass matrix $\mathbf{K}$ and the transmissivity matrix $\mathbf{T}$ which are both symmetric positive can be related to $L^{2}$ scalar products of elementary mapping problems to be solved on each fracture domains independently of each other. In practice, truncating the above equations with $n=1$ and $m=1$ corresponds to building a so called pipe network model characterized by uniform pressure at the intersections. The model was successfully implemented for 3D DFN [42].

In the present paper, we generalize the method in order to account for the matrix to fracture flow. We show that this can be achieved by changing the form of the mass term $\sum_{j \in J(i)} \sum_{n=1}^{\infty} K_{i j}^{m n} \times \frac{d P_{j}^{n}(t)}{d t}$ under the form of a time convolution involving the average transfer function $f(t)$ between the matrix and the fracture:

$$
\forall i=1, N_{n}, m=1, \infty, \quad \sum_{j \in J(i)} \sum_{n=1}^{\infty} K_{i j}^{m n} \times\left(V_{f} \delta(t)+V_{m} f(t)\right) * \frac{d P_{j}^{n}(t)}{d t}=\sum_{j \in J(i)} \sum_{n=1}^{\infty} V_{f} T_{i j}^{m n} \times P_{j}^{n} .
$$

The * symbol corresponds to a time convolution. $V_{f}$ and $V_{m}$ represent the volumic fractions of the fractures and of the matrix, $V_{f}+V_{m}=1$. The transfer function $f(t)\left[t^{-1}\right]$ appears as a time variable porosity. This function can be estimated by solving boundary value problem on the matrix blocks, or by alternative continuous time random walk 
60

62 in Laplace domain.

63

64

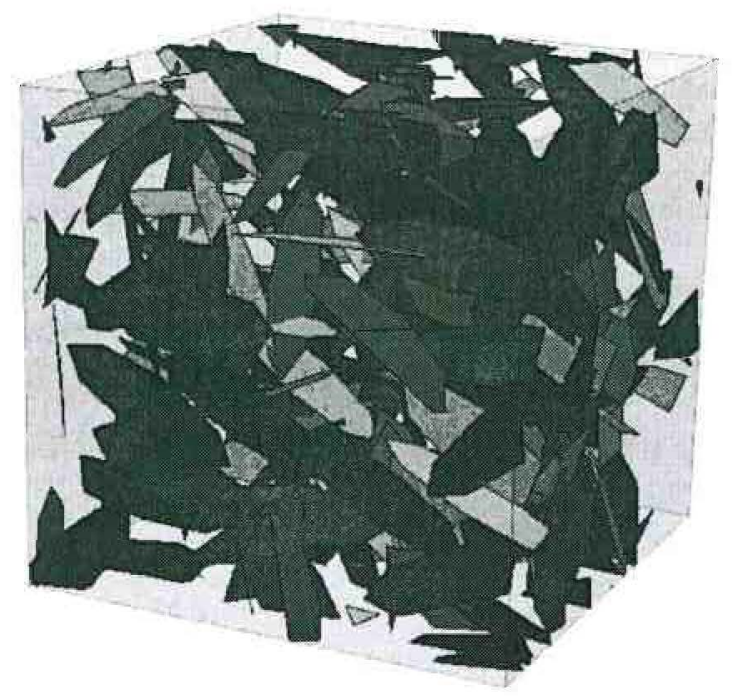

FIG. 1: $3 D$ network of $2 D$ polygonal fractures in a cubic box $\Omega$.

We consider flow in a $3 D$ cubic domain $\Omega$ containing $N$ distinct permeable fractures (FIG. 1 ) embedded in a low 76 permeability matrix. No flux boundary conditions will be considered first at the frontier of $\Omega$. The individual $I^{\text {th }}$ 
77 fracture is considered as being a closed $2 D$ object (e.g. polygonal or elliptic), the position of which can be given by

78 the coordinates of its center, the orientation of its normal, and all the necessary parameters chosen by the geologist

79 to characterize its detailed shape.

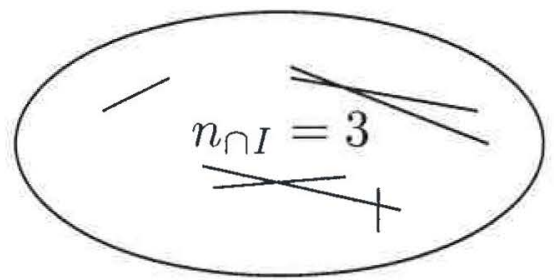

FIG. 2: An example of $2 D$ elliptic fracture with a number of "cluster of intersections" $n_{\cap I}=3$.

80 We consider a well connected network of $N$ fractures, so each fracture is connected to all the others via at least one

${ }_{81}$ path. So each fracture intersects at least one other fracture. Let $n_{\cap I}$ denotes the number of disconnected cluster of

${ }_{82}$ intersections of the $I^{\text {th }}$ fracture with the others. By the name "cluster of intersections", we mean that intersections

${ }_{83}$ between different fractures can intersect between each other (see FIG. 2 ), providing clusters that are not necessarily restricted as segments.

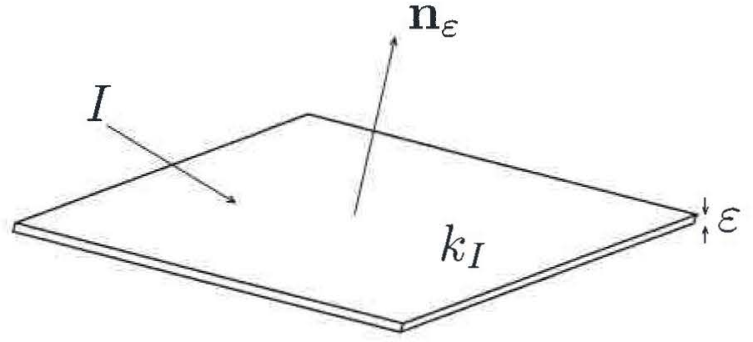

FIG. 3: $3 D$ fracture of thickness $\varepsilon$ and of permeability $k_{I}$.

84

On the hydrodynamic point of view, we consider that all the fractures share a small common thickness denoted by $\varepsilon$ (FIG. 3). The permeability of the $I^{\text {th }}$ fracture is denoted by $k_{I}$. This permeability may vary on the fracture's plane, but in order to simplify notations, this dependence will not be explained, although it will be accounted for in the $\nabla$ operator manipulations.

Our main goal is to study the solution of the following diffusion problem when $\varepsilon$ is small of:

$$
\begin{array}{r}
\varphi \mu c_{t} \frac{\partial p_{\varepsilon}(\mathbf{r}, t)}{\partial t}=\nabla \cdot\left(k(\mathbf{r}) \nabla p_{\varepsilon}(\mathbf{r}, t)\right)+g(\mathbf{r}) . \\
k(\mathbf{r})=k_{I} i f \mathbf{r} \in \Omega_{I} \text { forsomeI }=1, \ldots N \\
k(\mathbf{r})=k_{m} \text { else }
\end{array}
$$


The source term $g(\mathbf{r})$ is arbitrary for the moment: it can be a bulk source term. Here, we do not have to add boundary conditions at the fractures boundaries in contact with the matrix, but we may recall a normal flux continuity condition that will be ensured :

$$
\left(k_{I} \nabla p_{\varepsilon}(\mathbf{r}, t)\right) \cdot \mathbf{n}_{\varepsilon}=\left(k_{m} \nabla p_{\varepsilon}(\mathbf{r}, t)\right) \cdot \mathbf{n}_{\varepsilon}
$$

We denote by $D_{f}$ and $D_{m}$ the associated diffusion coefficients.

$$
\begin{gathered}
D_{f}=\frac{k_{I}}{\varphi \mu c_{t}} \\
D_{m}=\frac{k_{m}}{\varphi \mu c_{t}} \\
D_{m} \ll D_{f}
\end{gathered}
$$

Here $\varphi$ is the porosity and $c_{t}$ is the compressibility of the fluid, both are supposed to share the same value between matrix and fractures, an hypothesis that can be easily relaxed. $\mu$ is the fluid viscosity. Finally, in order to obtain a well-posed evolution problem, we assume that initial value data at $t=0$ are provided.

\section{THE PROJECTION FORMALISM}

\subsection{The projection formalism in the case of an impervious matrix}

In that section, we recall the results obtained in [1]. We consider first the steady state problem corresponding to the long time limit of 3 with an impervious matrix $k_{m}=0$. More details are given in the appendix A.

$$
\nabla \cdot\left(k_{I} \nabla p_{\varepsilon}(\mathbf{r})\right)=g_{I}(\mathbf{r}),
$$

The source term $g_{I}(\mathbf{r})$ corresponds to the restriction of $g(\mathbf{r})$ in the I th fracture domain. Notice that the Neumann' boundary conditions at the frontier of $\Omega$, and the well connectivity of the network, give a perfectly well posed problem in the fracture domain as far as the thickness $\varepsilon$ is not equal to zero. In [1], is was shown that the solution of Laplace equation in the fractured domain can be reconstructed once the trace of the pressure at the intersections between fractures is known. These intersections are generally segments. This trace may be in turn decomposed by projection on a complete set of basic function $\mathrm{A}$. The $\mathrm{n}$-th components of pressure on the $\mathrm{j}$ th intersection is denoted by $P_{j}^{n}$. In 
order to determine $P_{j}^{n}$, one needs boundary conditions at every intersection. It was shown that a correct boundary condition is that the sum of the (generally four) fluxes converging at a given point of the considered intersection is equal to zero (see appendix A and [1]). Projecting thus this condition of the same set of basis function gives thus the following linear system:

$$
\forall i=1, N_{\cap} ; m=1, \infty, \sum_{j \in J(i)} \sum_{n=1}^{\infty} T_{i j}^{m n} \times P_{j}^{n}=B_{i}^{m},
$$

Explicit expressions for $T_{i j}^{m n}$ are given in the appendix A. The right hand side $B_{i}^{m}$ are linear forms involving the source term $g_{I}$ are also given in the same appendix.

\subsection{Generalization to the transient case: the quasi steady state approximation}

The projection formalism can be adapted in order to solve the transient diffusion equation 3 with a source term in the fracture domain only. The proposed expression is a faithful approximation if the characteristic diffusion time over one fracture (of typical value $\tau \simeq \varphi \mu c_{t} L^{2} / k_{f} \ll \tau_{g_{I}}$ ) is smaller than the characteristic time of variation of the source term. this hypothesis is not restrictive at all and may be fulfilled in most practical cases. In that situation, the latter can appear as being stationary. The net result is a generalization of 9 that reads:

$$
\forall i=1, N_{n} ; m=1, \infty, \quad \sum_{j \in J(i)} \sum_{n=1}^{\infty} K_{i j}^{m n} \times \frac{d P_{j}^{n}(t)}{d t}=\sum_{j \in J(i)} \sum_{n=1}^{\infty} T_{i j}^{m n} \times P_{j}^{n}+B_{i}^{m} .
$$

The notations are essentially the same. This set of first order differential equations may be solved once an initial condition is fulfilled.

Explicit evaluation methods of $T_{i j}^{m n}$ and $K_{i j}^{m n}$ are given in the appendix $\mathrm{A}$ and B. Both matrices $\mathbf{K}$ and $\mathbf{T}$ are symmetric positive.

$$
\begin{aligned}
T_{i j}^{m n} & =T_{j i}^{n m}, \\
K_{i j}^{m n} & =K_{j i}^{n m},
\end{aligned}
$$

for all labels $i, j, m$ and $n$. 
We are now in position to couple the fracture network with the matrix. In order to fix the ideas, we solve the initial value problem 3. The initial value data at $t=0$ is $p(\mathbf{r}, \mathbf{t}=\mathbf{0})=\mathbf{0}$ if $\mathbf{r} \in$ matrix. Our first assumption is to consider than the ratio of typical diffusion time over an elementary matrix block having a characteristic size of $L$ (that can be considered as of the same order of magnitude of a fracture length) over a characteristic diffusion time over a single fracture that can be estimated as $\frac{k_{f}}{k_{m}}$ is very large. So, the pressure inside the blocks can be considered as slowly varying in the time domain. This observation permits us to use the preceding projection formalism at quasi steady state. At a given time and at a given location $\mathbf{r}$ inside a fracture, say the $I$-th fracture, one can compute a matrix to fracture flux $f_{I m f}(\mathbf{r}, \mathbf{t})$ given by

$$
f_{I m f}(\mathbf{r}, \mathbf{t})=\frac{k_{m}}{\mu}\left(\nabla p_{m}^{+}(\mathbf{r}, \mathbf{t})-\nabla \mathbf{p}_{\mathbf{m}}^{-}(\mathbf{r}, \mathbf{t})\right) \cdot \mathbf{n}_{\mathbf{I}}
$$

Here, $\mathbf{n}_{\mathrm{I}}$ is a vector normal to the $I$ th fracture, and the + or - signs correspond to both sides of the fracture. At

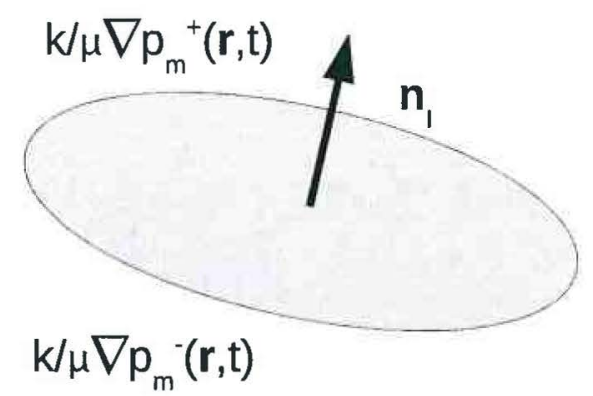

FIG. 4: Notations for the exchange flux

this stage, one must be careful with the small thickness $\varepsilon$ of the fracture, by considering as an intermediate step the full 3D diffusion problem 3 involving both fracture and matrix. In order to get meaningful results, we impose that 
${ }_{137} \quad k_{f} \times \frac{\left|\Omega_{f}\right|}{|\Omega|}>>k_{m}$, that states that the overall permeability of the fracture network dominates the matrix permeability.

${ }_{138}$ Since the fracture volume $\left|\Omega_{f}\right|$ is proportional to the fracture thickness $\varepsilon$, the limit $\varepsilon \rightarrow 0$ can causes some technical difficulties. Keeping $k_{f}$ constant and letting $\varepsilon \rightarrow 0$ should lead to a vanishing influence of the fractures, that is not of interest for our purposes. So, in the present situation in which we want to account for the matrix, it is more adapted

${ }_{141}$ to consider that the overall fracture conductivity proportional to $k_{f} \times \varepsilon$ is maintained fixed as $\varepsilon$ tends to zero.

142 In order to decouple the fracture and matrix problems, one can integrate the local equation over the 3D volume $V_{\varepsilon}$ of

143 thickness $\varepsilon$ bounded by a surface $\partial V_{\varepsilon}=S_{\varepsilon} \cup S_{+} \cup S_{-}$, as shown in FIG. 5 . The surface $S_{\varepsilon}$ is thus essentially a narrow band of thickness $\varepsilon$. One obtains:

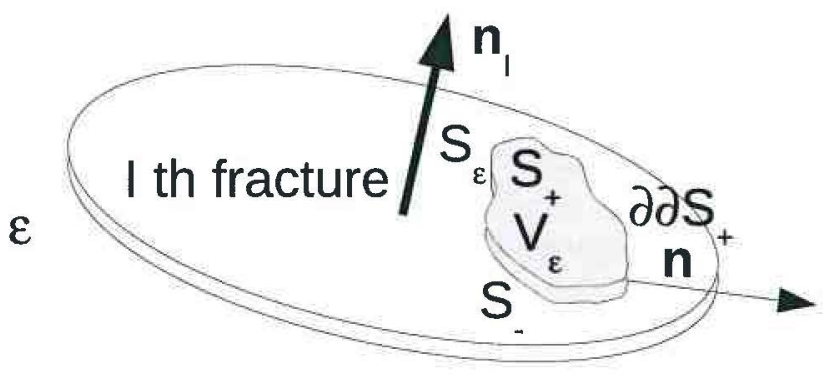

FIG. 5: Arbitrary integration domain $V_{\varepsilon}$ over the I th fracture

144

$$
\int_{V_{\varepsilon}} d^{3}(\mathbf{r}) \varphi \mu c_{t} \frac{\partial p_{\varepsilon}(\mathbf{r}, t)}{\partial t}=\int_{V_{\varepsilon}} d^{3}(\mathbf{r}) \nabla \cdot\left(k(\mathbf{r}) \nabla p_{\varepsilon}(\mathbf{r}, t)\right)
$$

${ }_{145}$ Using Green's theorem on the right hand side of this equation, one obtains:

$$
\int_{V_{\varepsilon}} d^{3} \mathbf{r} \varphi \mu c_{t} \frac{\partial p_{\varepsilon}(\mathbf{r}, t)}{\partial t}=\int_{\partial V_{\varepsilon}} d^{2} \mathbf{r n} \cdot\left(k(\mathbf{r}) \nabla p_{\varepsilon}(\mathbf{r}, t)\right)
$$


So, using the decomposition $\partial V_{\varepsilon}=S_{\varepsilon} \cup S_{+} \cup S_{-}$, one gets

$$
\int_{V_{\varepsilon}} d^{3} \mathbf{r} \varphi \mu c_{t} \frac{\partial p_{\varepsilon}(\mathbf{r}, t)}{\partial t}=\int_{\partial S_{\varepsilon}} d^{2} \mathbf{r n} \cdot\left(k(\mathbf{r}) \nabla p_{\varepsilon}(\mathbf{r}, t)\right)+\int_{\partial S_{+}} d^{2} \mathbf{r} f_{I m f}(\mathbf{r}, \mathbf{t})
$$

One can exploit the smallness of $\varepsilon$ to estimate the various integrals and in order to decrease the order of integration.

$$
\varepsilon \int_{S_{+}} d^{2} \mathbf{r} \varphi \mu c_{t} \frac{\partial p_{\varepsilon}(\mathbf{r}, t)}{\partial t} \simeq \varepsilon \int_{\partial \partial S_{+}} d \mathbf{r n} \cdot\left(k(\mathbf{r}) \nabla p_{\varepsilon}(\mathbf{r}, t)\right)+\int_{\partial S_{+}} d^{2} \mathbf{r} f_{I m f}(\mathbf{r}, \mathbf{t})
$$

$$
g_{I}(\mathbf{r})=\frac{1}{\varepsilon} f_{I m f}(\mathbf{r}, \mathbf{t})
$$

The $2 D$ integration was transformed into a $1 D$ one along the curve denoted by $\partial \partial S_{+}$. The normal vector $\mathbf{n}$ is the normal vector to the $1 \mathrm{D}$ curve $\partial \partial S_{+}$belonging to the I th fracture plane. As the preceding equality is exact for any $\partial \partial S_{+}$, and using Green's theorem, one gets the local $2 D$ equation valid only on the fracture plane:

$$
\varepsilon \varphi \mu c_{t} \frac{\partial p_{\varepsilon}(\mathbf{r}, t)}{\partial t}=\varepsilon \nabla \cdot\left(k(\mathbf{r}) \nabla p_{\varepsilon}(\mathbf{r}, t)\right)+f_{I m f}(\mathbf{r}, \mathbf{t})
$$

In present form, the limit $\varepsilon \rightarrow 0$ can be evaluated safely. The differential operators are defined as in the preceding sections on the considered fracture only. In practice, we have essentially to solve the same equations, up to a factor $\varepsilon$. The projection formalism can be used on the fracture domain using the following correspondence:

The next issue will be to relate the interporosity flux $f_{\operatorname{Imf}}(\mathbf{r}, \mathbf{t})$ to the fracture pressure $p_{\varepsilon}(\mathbf{r}, t)=p(\mathbf{r}, t)$. The subscript $\varepsilon$ can be suppressed now to simplify notations.

\subsection{About the matrix to fracture flow, the exchange function}

In order to specify a workable form of $f_{\operatorname{Imf}}(\mathbf{r}, \mathbf{t})$, a possible option is to relate $f_{\operatorname{Imf}}(\mathbf{r}, \mathbf{t})$ to the pressure map $p_{I}(\mathbf{r}, \mathbf{t})$ of the I th fracture. The basic assumption is to consider a time convolution form that keeps the causality and linearity of the underlying equations:

$$
\frac{k_{m}}{\mu} \nabla p_{m}^{+}(\mathbf{r}, \mathbf{t}) \cdot \mathbf{n}_{\mathbf{I}}=-\int_{0}^{\mathbf{t}} \mathbf{d} \mathbf{t}^{\prime} \mathbf{f}_{\mathrm{Im}}^{+}\left(\mathbf{t}-\mathrm{t}^{\prime}\right) \frac{\partial \mathbf{p}_{\mathbf{I}}\left(\mathbf{r}, \mathbf{t}^{\prime}\right)}{\partial \mathbf{t}^{\prime}}
$$




$$
g_{I}(\mathbf{r})=-\frac{1}{\varepsilon} \int_{0}^{t} d t^{\prime} f_{I m}\left(t-t^{\prime}\right) \frac{\partial p_{I}\left(\mathbf{r}, \mathbf{t}^{\prime}\right)}{\partial t^{\prime}}
$$

This form may be justified by the following arguments: let $\Omega_{m}$ be a matrix block surrounded by several fractures. Let $P_{f}(t)$ be the pressure of these fractures, assumed to be spatially uniform. We consider a solution of the diffusion equation inside the matrix block without any source term:

$$
\begin{array}{r}
\varphi \mu c_{t} \frac{\partial p_{m}(\mathbf{r}, t)}{\partial t}=\nabla \cdot\left(k_{m} \nabla \delta p_{m}(\mathbf{r}, t)\right)=0 \\
p_{m}(\mathbf{r}, t)=P_{f}(t) \quad \text { if } \quad \mathbf{r} \in \partial \Omega_{m} \\
\forall \mathbf{r} \in \Omega_{m}, p_{m}(\mathbf{r}, t=0)=0
\end{array}
$$

The present goal is to relate the flux $\frac{k_{m}}{\mu} \nabla p_{m}^{+}(\mathbf{r}, \mathbf{t}) \cdot \mathbf{n}$, or at least its average $\frac{1}{\left|\partial \Omega_{m}\right| ?} \int_{\partial \Omega_{m}} d^{2} \mathbf{r} \frac{k_{m}}{\mu} \nabla p_{m}^{+}(\mathbf{r}, \mathbf{t}) \cdot \mathbf{n}$ to the variations of the pressure at the boundary $P_{f}(t)$. Applying the divergence theorem, we obtain:

$$
\int_{\partial \Omega_{m}} d^{2} \mathbf{r} \frac{k_{m}}{\mu} \nabla p_{m}^{+}(\mathbf{r}, \mathbf{t}) \cdot \mathbf{n}=-\varphi \mathbf{c}_{\mathbf{t}}\left|\Omega_{\mathbf{m}}\right| \frac{\mathbf{d}\left\langle\mathbf{P}_{\mathbf{m}}\right\rangle(\mathbf{t})}{\mathbf{d t}}=-\varphi \mathbf{c}_{\mathbf{t}} \frac{\mathbf{d} \int_{\Omega_{\mathbf{m}}} \mathbf{d}^{3} \mathbf{r} \mathbf{P}_{\mathbf{m}}(\mathbf{r}, \mathbf{t})}{\mathbf{d t}}
$$

Here $\langle\cdots\rangle$ denotes a volume average of the pressure $P_{m}$ over the matrix block. The minus sign comes from the normal $\mathrm{n}$ orientation.

It is now possible to search for a relation between $\left\langle P_{m}\right\rangle(t)$ and $P_{f}(t)$ under the form of a convolution product:

$$
\left\langle P_{m}\right\rangle(t)=\int_{0}^{t} d t^{\prime} f\left(t-t^{\prime}\right) P_{f}\left(t^{\prime}\right)
$$

The mapping function $f(t)$, homogeneous to an inverse of time is the solution of a well posed boundary value problem that will be discussed in more details in 5. Coming back to the average flux, using 24 and 25, and elementary properties of convolution products, we get the following form:

$$
\int_{\partial \Omega_{m}} d^{2} \mathbf{r} \frac{k_{m}}{\mu} \nabla p_{m}^{+}(\mathbf{r}, \mathbf{t}) \cdot \mathbf{n}=-\varphi \mathbf{c}_{\mathbf{t}}\left|\Omega_{\mathbf{m}}\right| \int_{\mathbf{0}}^{\mathbf{t}} \mathbf{d t ^ { \prime }} \mathbf{f}\left(\mathbf{t}-\mathbf{t}^{\prime}\right) \frac{\mathrm{dP}_{\mathbf{f}}\left(\mathbf{t}^{\prime}\right)}{\mathbf{d t}^{\prime}},
$$




$$
\frac{k_{m}}{\mu} \nabla p_{m}^{+}(\mathbf{r}, \mathbf{t}) \cdot \mathbf{n}_{\mathbf{I}}=-\int_{0}^{\mathbf{t}} \mathbf{d t}^{\prime} \varphi \mathbf{c}_{\mathbf{t}} \frac{\left|\Omega_{\mathbf{m}}\right|}{\left|\partial \Omega_{\mathbf{m}}\right|} \mathbf{f}^{+}\left(\mathbf{t}-\mathbf{t}^{\prime}\right) \frac{\partial \mathbf{p}_{\mathbf{I}}\left(\mathbf{r}, \mathbf{t}^{\prime}\right)}{\partial \mathbf{t}^{\prime}}
$$

Coming back to the local interporosity flux $\frac{k_{m}}{\mu} \nabla p_{m}^{+}(\mathbf{r}, \mathbf{t}) \cdot \mathbf{n}_{\mathbf{I}}$ in the general case of a non uniform pressure in the fractures, the preceding developments suggest the proposed form that leads to 21 .

$$
\begin{aligned}
g_{I}(\mathbf{r}) & =-\varphi c_{t} \frac{\left|\Omega_{m}\right|}{\varepsilon\left|\partial \Omega_{m}\right|} \int_{0}^{t} d t^{\prime} f\left(t-t^{\prime}\right) \frac{\partial p_{I}\left(\mathbf{r}, \mathbf{t}^{\prime}\right)}{\partial t^{\prime}} \\
& =-\varphi c_{t} \frac{V_{m}}{V_{f}} \int_{0}^{t} d t^{\prime} f\left(t-t^{\prime}\right) \frac{\partial p_{I}\left(\mathbf{r}, \mathbf{t}^{\prime}\right)}{\partial t^{\prime}}
\end{aligned}
$$

A more detailed discussion about the $f(t)$ functions will be provided in section 5 . We now turn our attention about the final closure of the problem, and the resulting consequences on the projection formalism.

\subsection{Final closure and projection formalism}

Combining 3 and 28 , we get an equation driving $p(\mathbf{r}, t)$ inside any fracture:

$$
\varphi \mu c_{t} \frac{\partial p(\mathbf{r}, t)}{\partial t}=\nabla \cdot\left(k_{I} \nabla p(\mathbf{r}, t)\right)-\frac{V_{m}}{V_{f}} \varphi \mu c_{t} \int_{0}^{t} d t^{\prime} f\left(t-t^{\prime}\right) \frac{\partial p\left(\mathbf{r}, \mathbf{t}^{\prime}\right)}{\partial t^{\prime}}
$$

When it is possible, we suppress the subscript $I$ because we are considering local pressure without any ambiguity on the I th fracture. As the local pressure $p(\mathbf{r}, t)$ appears in both members, the original diffusion equation becomes an integral equation which can be rewritten under the alternative form:

$$
\int_{0}^{t} d t^{\prime} \varphi \mu c_{t}\left(V_{f} \delta\left(t-t^{\prime}\right)+V_{m} f\left(t-t^{\prime}\right)\right) \frac{\partial p\left(\mathbf{r}, t^{\prime}\right)}{\partial t^{\prime}}=V_{f} \nabla \cdot\left(k_{I} \nabla p(\mathbf{r}, t)\right)
$$


As in [1], we introduce the following pressure decomposition, see appendices A and B for the details.

$$
p(\mathbf{r}, t)=\delta p(\mathbf{r}, t)+\sum_{j \in J_{I}} \sum_{n=1}^{\infty} P_{j}^{n}(t) \hat{P}_{j}^{n}(\mathbf{r})
$$

${ }_{184}$ The pressure fluctuation $\delta p(\mathbf{r}, t)$ follows the equation:

$$
\begin{aligned}
\varphi \mu c_{t} \int_{0}^{t} d t^{\prime}\left(V_{f} \delta\left(t-t^{\prime}\right)+V_{m} f\left(t-t^{\prime}\right) \frac{\partial \delta p\left(\mathbf{r}, t^{\prime}\right)}{\partial t}\right. & =V_{f} \nabla \cdot\left(k_{I} \nabla \delta p(\mathbf{r}, t)\right) \\
& -\sum_{j \in J_{I}} \sum_{n=1}^{\infty} \int_{0}^{t} \varphi \mu c_{t}\left(V_{f} \delta\left(t-t^{\prime}\right)+V_{m} f_{I m}\left(t-t^{\prime}\right)\right) \dot{P}_{j}^{n}\left(t^{\prime}\right) \hat{P}_{j}^{n}(\mathbf{r}),(30)
\end{aligned}
$$

185 with an additional condition:

$$
\delta p(\mathbf{r}, t)=0 \quad \text { if } \quad \mathbf{r} \in \cup \cap_{i}
$$

186 Here, the $P_{j}^{n}(t)$ are assumed to be "slowly varying" if compared to typical diffusion time over one fracture. The steady state assumption assumes that the residual term $\delta p(\mathbf{r}, t)$ obeys a steady state equation in which the left hand side is considered as negligible. The validity of this major assumption will be discussed in more details in section 6 . So, we assume that

$$
V_{f} \nabla \cdot\left(k_{I} \nabla \delta p(\mathbf{r}, t)\right)=\sum_{j \in J_{I}} \sum_{n=1}^{\infty} \int_{0}^{t} \varphi \mu c_{t}\left(V_{f} \delta\left(t-t^{\prime}\right)+V_{m} f\left(t-t^{\prime}\right)\right) \dot{P}_{j}^{n}\left(t^{\prime}\right) \hat{P}_{j}^{n}(\mathbf{r})
$$

We can combine B2 and 19 to get:

$$
\forall i, m, \quad \sum_{j \in J(i)} \sum_{n=1}^{\infty} K_{i j}^{m n} \times \int_{0}^{t}\left(V_{f} \delta\left(t-t^{\prime}\right)+V_{m} f_{I m}\left(t-t^{\prime}\right)\right) \frac{d P_{j}^{n}\left(t^{\prime}\right)}{d t}=\sum_{j \in J(i)} \sum_{n=1}^{\infty} V_{f} T_{i j}^{m n} \times P_{j}^{n}
$$
192 by

$$
g(s)=\int_{0}^{\infty} \exp -\operatorname{stg}(t) d t
$$

193 With the property for the Laplace transform of the time derivative of a function $g(t)$ : 


$$
\left[\frac{d g(t)}{d t}\right](s)=s g(s)-g(t=0)
$$

$$
\forall i, m, \quad \sum_{j \in J(i)} \sum_{n=1}^{\infty} K_{i j}^{m n} \frac{d P_{j}^{n}}{d t} \simeq \sum_{j \in J(i)} \sum_{n=1}^{\infty} V_{f} T_{i j}^{m n} \times P_{j}^{n}(t)
$$

$$
\forall i, m, \quad \sum_{j \in J(i)} \sum_{n=1}^{\infty} K_{i j}^{m n} \times\left(V_{f}+V_{m} f(s)\left(s P_{j}^{n}(s)-P_{j}^{n}(t=0)\right)=\sum_{j \in J(i)} \sum_{n=1}^{\infty} V_{f} T_{i j}^{m n} \times P_{j}^{n}(s)\right.
$$

In the Laplace domain, the net effect of the matrix is a modification of the porosity by a $s$ dependent porosity. Setting $V_{m}=0$, one recovers the impervious matrix case equations 1. It appears that having a solution of the corresponding impervious matrix problem given by 1 using Laplace transforms, and replacing the Laplace argument $s$ by $s\left(V_{f}+V_{m} f(s)\right)$ will provide the solution of 34. Numerical Laplace inversion can be performed with accuracy by Stehfest algorithm [45]. The net result is that using Laplace transform techniques, the additional computational cost relies mainly in the determination of the exchange function $f($.$) . This observation was already highlighted by$ several authors in the context of the averaged continuous double porosity descriptions with transient interporosity flow $[24-26]$.

Finally, for small $s$, as $f(s) \sim 1$ (section 5 ) corresponding to long time relaxation or low frequencies forcing, one obtains, coming back to the time domain:

This corresponds to the original set of equations, up to a $V_{f}$ factor that appears as a retention factor that will hinder diffusion in the fracture domain. 
We can now study the exchange function as well as its practical evaluation. We recall 25 relating the volume average

$\left\langle P_{m}\right\rangle(t)=$ of the pressure in the matrix to the forcing imposed by the boundary condition in the fractures $P_{f}(t)$ :

$$
\left\langle P_{m}\right\rangle(t)=\int_{0}^{t} d t^{\prime} f\left(t-t^{\prime}\right) P_{f}\left(t^{\prime}\right)
$$

Or, equivalently using Laplace transforms:

$$
\left\langle P_{m}\right\rangle(s)=f(s) P_{f}(s)
$$

Choosing as $P_{f}(t)$ a Heaviside function gives: $\left\langle P_{H m}\right\rangle(t)=\int_{0}^{t} d t^{\prime} f\left(t^{\prime}\right)$, from which $f(t)$ can be obtained by direct time derivative evaluation. So, solving the following boundary value problem:

$$
\begin{array}{r}
\varphi \mu c_{t} \frac{\partial p_{H m}(\mathbf{r}, t)}{\partial t}=\nabla \cdot\left(k_{m} \nabla \delta p_{H m}(\mathbf{r}, t)\right), \\
p_{m}(\mathbf{r}, t)=1 \quad \text { if } \quad \mathbf{r} \in \partial \Omega_{m}, t>0 \\
p_{m}(\mathbf{r}, t=0)=0 \quad \forall \mathbf{r} \in \Omega_{m}
\end{array}
$$

and computing the average $\left\langle P_{H m}\right\rangle(t)$ yields the exchange function. This evolution equation can be solved by several numerical methods. The main task is to mesh the matrix. Note that any explicit meshing of the fractures is avoided because the fractures enter only via a Dirichlet boundary condition. The resulting linear systems to be solved will not contain highly contrasted coefficients, because $k_{f}$ does not enter in the problem, so correct preconditioning properties can be expected. A useful alternative interpretation of the exchange function in terms of random walks can be proposed [20,22]. Alternative numerical techniques such as MINC approaches can also be employed [37].

\subsection{Properties of the exchange function}

Equations 25 and 39 permits to obtain some analytical solutions in simple cases for $f(t)$ or $f(s)$. We can consider D blocks (the associated coordinate $x \in[0,2 \ell]$ perpendicular to the plane of the fracture). The potential $p_{H m}(x, t)$ 
depends on $x$ and $t$, So one can use the $1 D$ solution:

$$
\begin{array}{r}
\frac{\partial p_{H m}(x, t)}{\partial t}=D_{m} \frac{\partial^{2} p_{H m}(x, t)}{\partial x^{2}}, \\
p_{m}(x=0, t)=1 \text { for } t>0 \\
\frac{\partial p_{H m}}{\partial x}(x=\ell, t)=0, \text { for } t>0 \text { by symmetry } \\
\forall x \neq 0, p_{m}(x, t=0)=0
\end{array}
$$

this equation can be solved using time domain Laplace transform:

$$
\begin{array}{r}
s p_{H m}(x, s)=D_{m} \frac{\partial^{2} p_{H m}(x, s)}{\partial x^{2}}, \\
p_{m}(x=0, s)=1 / s \\
\frac{\partial p_{H m}}{\partial x}(x=\ell, s)=0, \text { for } t>0 \text { by symmetry }
\end{array}
$$

This single variable differential equation can be solved easily. One obtains finally:

$$
f(s)=\frac{\sqrt{D_{m}}}{\ell \sqrt{s}} \times \operatorname{th}\left(\sqrt{\frac{s}{D_{m}}} \ell\right)
$$

At short times $t$, when the potential in the fractures corresponding to the boundaries of the matrix blocks, is set to 1, the diffusion in the matrix takes place only in a small boundary layer close to the fractures. One can adopt two point of views, in the first one, one can write $f(s)=\frac{\sqrt{D_{m}}}{\ell \sqrt{s}} \times t h\left(\sqrt{\frac{s}{D_{m}}} \ell\right) \simeq \frac{\sqrt{D_{m}}}{\ell \sqrt{s}}$. In the second point of view, one can consider that the matrix blocks are infinite, $\ell=\infty$, which does not permit using 43 directly because the average pressure on the matrix is not well defined. But, one can use directly the evaluation 20 of the matrix to fracture flux.

$$
\begin{array}{r}
s p_{H m}(x, s)=D_{m} \frac{\partial^{2} p_{H m}(x, s)}{\partial x^{2}}, \\
p_{m}(x=0, s)=1 / s
\end{array}
$$


and to compute $\varphi c_{t} D_{m} \frac{\partial p_{H m)}}{\partial x}(x=0, s)$ for $x>0$ by symmetry to obtain:

$$
f(s)=\frac{\sqrt{D_{m}}}{\sqrt{s}} \times \frac{\left|\partial \Omega_{m}\right|}{\left|\Omega_{m}\right|}
$$

234 In the real time domain, this corresponds to

$$
f(t)=\frac{\sqrt{D_{m}}}{\sqrt{\pi} \sqrt{t}} \times \frac{\left|\partial \Omega_{m}\right|}{\left|\Omega_{m}\right|}
$$

Comparing both results shows that for consistency, $\ell=\frac{\left|\Omega_{m}\right|}{\left|\partial \Omega_{m}\right|}$. This formula can be interpreted as follows: at short times $t$, the characteristic diffusion length is of the order of $\sqrt{D_{m} t}$. So the corresponding flux is given by 46 up to numerical constants. This corresponds to the large $s$ asymptotics of $f(s)$.

Several other general properties of $f(t)$ can be attained by studying the limit $s \rightarrow 0$. One has, using a Taylor expansion of the th function:

$$
f(s)=1-\frac{1}{3} \frac{s}{D_{m}} \ell^{2}+\ldots
$$

We observe that $f(s=0)=1$. This is a general equality that occurs because using 36 and remarking that both fracture and matrix potential equalize at the long times (or low frequency) limit so $f(s=0)=1$. In next paragraph, we show that the linear term in $s$ is closely related to the so called "exchange coefficient" $\alpha_{\infty}=3 \frac{D_{m}}{V_{m} \ell^{2}}$ in present case. This coefficient arises from large scale averaging theories that yields homogenized form of double porosity equations [21] valid at long times, long distances. A useful interpretation of $f(t)$ in terms of escape time pdf from the matrix was derived in $[20,22]$. It corresponds to the exit time distribution from the matrix blocks of a particle undergoing brownian motion of diffusion coefficient $\frac{k_{m}}{\phi \mu c_{t}}$. In particular, the average exit time may be directly related to the so called exchange coefficient or "shape factor" that enter in classical dual porosity models $[17,21]$. Continuous time random walk techniques can thus be set-up to determine this exit time distribution. This can provide techniques avoiding any explicit meshing of the matrix. Detailed expressions of exchange functions using Laplace transforms are given for several block geometries by de Swann [23-25], that can be useful for testing numerical solutions or analytical parameterizations. 


$$
f(s) \approx \frac{\alpha_{\infty}}{V_{m} s+\alpha_{\infty}}
$$

corresponding to an exponential relaxation in the time domain is equivalent to consider a steady state double porosity model [22]. Using 48 and 24, it is possible to show that at a given time, the flux between matrix and the fractures is given by:

$$
\frac{k_{m}}{\mu} \nabla p_{m}(\mathbf{r}, \mathbf{t}) \cdot \mathbf{n}=\frac{\left|\Omega_{\mathbf{m}}\right|}{\left|\partial \Omega_{\mathbf{m}}\right|} \varphi \mathbf{c}_{\mathbf{t}} \alpha_{\infty}\left(\left\langle\mathbf{P}_{\mathbf{m}}\right\rangle(\mathbf{t})-\mathbf{P}_{\mathbf{I}}(\mathbf{t})\right)
$$

This corresponds to a steady state double porosity model with a particular choice of the so called shape factor $\alpha_{\infty}$ [17]. The flux is proportional to the difference between the pressure of the matrix and the fracture. The reader should note that in [22], the exchange function $f(s)$ corresponds to $V_{f}+V_{m} f(s)$.

\section{TESTING THE SELF CONSISTENCY OF THE ASSUMPTIONS}

In that section, we verify on a simplified test problem whether the quasi steady state assumption of section 44.3 is consistent with the subsequent findings. In other words, we check if the source term arising from the matrix does not modify drastically the pressure diffusion inside a fracture, that could lead to fracture relaxation time comparable with the matrix relaxation time. We come back about a simplified form of 30 on a single fracture, keeping our notations:

$$
\begin{gathered}
\varphi \mu c_{t} \int_{0}^{t} d t^{\prime}\left(V_{f} \delta\left(t-t^{\prime}\right)+V_{m} f\left(t-t^{\prime}\right) \frac{\partial \delta p\left(\mathbf{r}, t^{\prime}\right)}{\partial t}=V_{f} \nabla \cdot\left(k_{I} \nabla \delta p(\mathbf{r}, t)\right)\right. \\
\delta p(\mathbf{r}, t=0) \quad \text { fixed }
\end{gathered}
$$

It corresponds to an initial value problem on the fracture domain, without source term. We want to check if the relaxation time associated with the operator $\varphi \mu c_{t} \int_{0}^{t} d t^{\prime}\left(V_{f} \delta\left(t-t^{\prime}\right)+V_{m} f\left(t-t^{\prime}\right) \frac{\partial \delta p\left(\mathbf{r}, t^{\prime}\right)}{\partial t}\right.$ is small compared with the diffusion time in the matrix $\simeq D_{m} / \ell^{2}$. In order to proceed, we consider that $f(s)$ is given by the steady state double porosity model 48 . We replace also the Laplace operator $V_{f} \nabla \cdot\left(k_{I} \nabla \delta p(\mathbf{r}, t)\right)$ by its smallest eigenvalue corresponding to the larger relaxation time of the fracture: $V_{f} \nabla \cdot\left(k_{I} \nabla \delta p(\mathbf{r}, t)\right) \sim-V_{f} \varphi \mu c_{t} \lambda \delta p(\mathbf{r}, t)$. Here, $\lambda \sim D_{f} / \ell^{2} \gg \alpha_{\infty} \sim D_{m} / \ell^{2}$. 
equivalent to:

or, equivalently:

$$
\left(V_{f}+V_{m} \frac{\alpha_{\infty}}{V_{m} s+\alpha_{\infty}}\right) s \delta p(\mathbf{r}, s)=-V_{f} \lambda \delta p(\mathbf{r}, s)+V_{f} \delta p(\mathbf{r}, t=0)
$$

$$
\delta p(\mathbf{r}, s)=\frac{\left(V_{m} s+\alpha_{\infty}\right)}{\left(V_{f} V_{m} s^{2}+\left(\alpha_{\infty}+\lambda V_{f} V_{m}\right) s+V_{f} \lambda \alpha\right.} V_{f} \delta p(\mathbf{r}, t=0)
$$

Recalling the boundary conditions for $\delta p(\mathbf{r}, s)=0$ at the fractures intersections, it is clear that for large time $\delta p(\mathbf{r}, t) \rightarrow 0$ for $t \rightarrow \infty$. The convergence is exponential and in order to estimate the relaxation time, the roots of the denominator has to be evaluated. The resulting expressions can be simplified using the fact that $\alpha_{\infty} \ll \lambda$, and we obtain two roots, up to terms of order $\frac{\alpha_{\infty}}{\lambda} \ll 1$, which are $-\lambda$ and $-\frac{\alpha_{\infty}}{\varphi_{m}}$. Finally, after simplification with the numerator, we get:

$$
\delta p(\mathbf{r}, s)=\frac{1}{s+\lambda} \delta p(\mathbf{r}, t=0)
$$

$$
\delta p(\mathbf{r}, t)=\exp -(\lambda t) \delta p(\mathbf{r}, t=0)
$$

This confirms the fast relaxation of the transient and the justification of the quasi steady state approximation.

In that subsection, we restrict the problem to the case $m=1$ and $n=1$ used in practice [42]. In practice it means physically that we estimate only the average pressure along each intersection, and that the mass conservation equation at the intersection is only fulfilled globally. Assuming that all $P_{i}^{m}=0$ if $m \geq 2$, the differential system to be solved 284 is: 


$$
\forall i, \quad \sum_{j \in J(i)} K_{i j}^{11} \times\left(V_{f} \delta(t)+V_{m} f(t)\right) * \frac{d P_{j}^{1}(t)}{d t}=\sum_{j \in J(i)} V_{f} T_{i j}^{11} \times P_{j}^{1} .
$$

We recall the following relation [1]:

$$
\sum_{i j \in \Omega I} K_{i j}^{11}=\varphi \mu c_{t} \int_{\Omega_{I}} d^{2} \mathbf{r}=\varphi \mu c_{t} S_{I}
$$

where $S_{I}$ is the total area of the fracture.

The resulting time discretization scheme of the differential equations (51) can be rather time consuming in the case of large fracture networks. It is thus appealing to use a mass condensation (or mass lumping) scheme by acknowledging that pressure variations of neighboring nodes will be very close together. We replace $\sum_{j \in J(i)} K_{i j}^{11} \times$ $\frac{d P_{j}^{1}(t)}{d t}$ by $\sum_{j \in J(i)} K_{i j}^{11} \times \frac{d P_{i}^{1}(t)}{d t}$. So we get:

$$
\begin{aligned}
\forall i, \quad M_{i} \times\left(V_{f} \delta(t)+V_{m} f(t)\right) * \frac{d P_{i}^{1}(t)}{d t} & =\sum_{j \in J(i)} V_{f} T_{i j}^{11} \times\left(P_{j}^{1}-P_{i}^{1}\right), \\
\text { with } M_{i} & =\sum_{j \in J(i)} K_{i j}^{11} .
\end{aligned}
$$

Indeed, these equations (52) possess the structure of the equations driving the variations of the node potentials of a resistor/capacitor network. The main difference is that the capacity term appears under the form of convolution products, that are simple products in the Laplace domain.

The numerical determination of the $T_{i j}^{11}$ can be done by solving $n_{\cap}$ elementary Laplace problems with the boundary conditions at the intersections, and computing next the scalar products (A15). Thus, the masses $M_{i}$ given by (53) are obtained using a suitable surface integration scheme. Fast evaluations of these quantities avoiding solving local Laplace problems on each fracture are proposed in [42].

In this paper, we generalize a method that permits to solve diffusion problems in complex $3 D$ fracture networks using a relatively small number of degrees of freedom. The generalization enables us to consider flows coupled with a low permeability matrix acting as a reservoir. The flow exchanges with the matrix can be modeled using the so called 
exchange function $f($.$) . The main assumption is that diffusion is so fast in the fractures that the matrix blocks are$ bounded by essentially spatially uniform boundary conditions that are quasi steady state. The second assumption is to replace the local matrix to fracture flux by its average. Both assumptions permit to define $f($.$) as a volume$ average of a solution of a well posed boundary value problem. This function can be determined by existing numerical techniques that avoid a complex meshing of the DFN and solving a badly conditioned problem. It posses also a probabilistic interpretation as it represents the pdf of escape time of diffusing particle in the matrix. Alternatively, it can be parameterised using generic analytical forms fulfilling asymptotic requirements at short and long times. These forms permit to capture the essential features of the matrix: The surface to volume ratio, the typical size $L$ of the blocks, and a shape factor. Very ramified DFN with many dead ends having fractal like structures could be described using a power law $f($.$) function accounting for scale dependent surface to volume ratio. Using Laplace transform$ techniques, we show that the effect of the matrix can be modeled at a small extra cost once a previous modeling of potential diffusion in the DFN with an impervious matrix is available. No major extra computing cost can be expected. Numerical tests have to be carried out in order to test the accuracy of the approach, and especially of the limitation to the pipe network approximation $(\mathrm{n}, \mathrm{m})$ restricted to 1 . Another issue is the generalization of the present formalism to other transport equations, such as convection diffusion equations in the fracture network, coupled with purely diffusive transport in the matrix. This could be done following works of [27] and [28].

\section{APPENDIX A: PROJECTION FORMALISM STEADY STATE CASE}

\section{Small fracture thickness limit}

The projection method follows several steps. The first one is to account for the small thickness $\varepsilon$ of the fractures in order to be able to treat the intersection between fractures as $1 D$ objects, and the fractures as $2 D$ objects embedded in a $3 D$.

Some geometrical quantities and several notations are presented in (FIG. 6). Let $\cap_{I J}$ be an intersection between the two fractures $I$ and $J$; a $3 D$ volume having the shape of a match. As $\varepsilon$ tends to zero, this volume becomes a $1 D$ segment corresponding to the intersection of the two planes containing fractures $I$ and $J$. In order to simplify the discussion, and this changes nothing to the global solution, we consider that this segment does not intersect a third fracture. Let $x$ denote a coordinate along this segment. The point $\mathbf{r}_{\cap_{I J}}(x)$ denotes in a rather natural way the generic point of this segment labeled by $x$ (in practice, the three coordinates of $\mathbf{r}_{\cap_{I J}}(x)$ may depend linearly on $x$ ). 

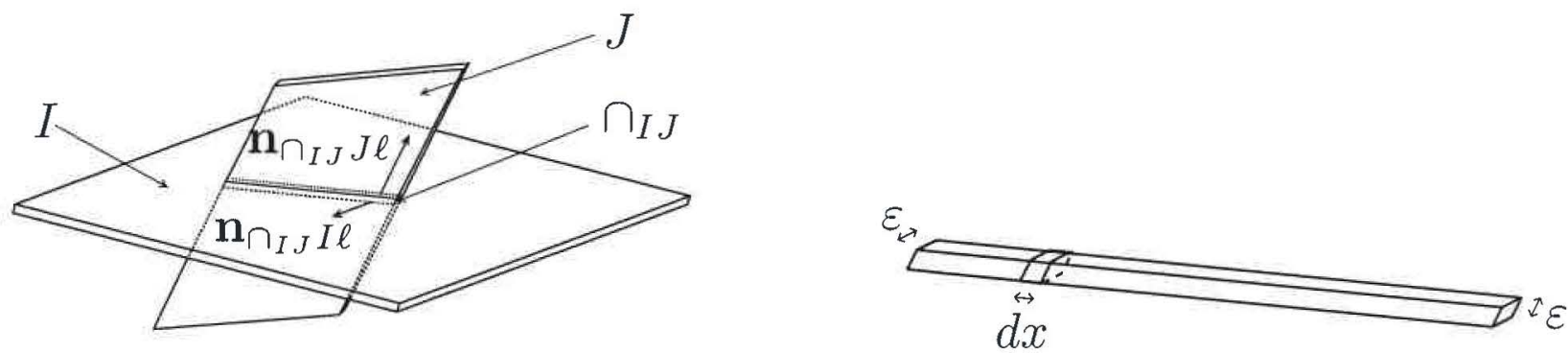

$\downarrow \varepsilon \rightarrow 0$
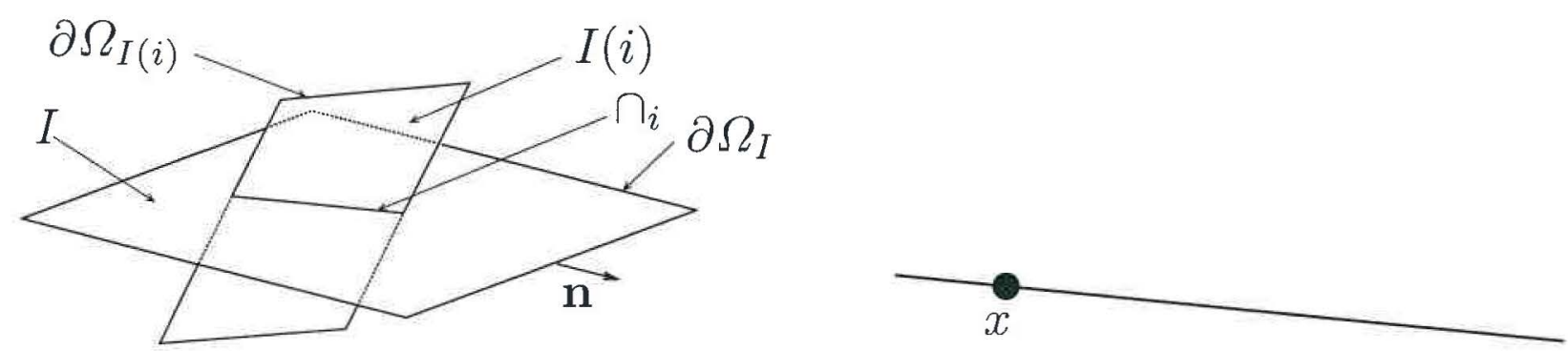

FIG. 6: Geometry and notation two rectangular fractures and the associated intersection, and limit $\varepsilon \rightarrow 0$.

As $\varepsilon$ tends to zero, we can consider that close to the point $\mathbf{r}_{\cap_{I J}}(x)$, the intersection separates locally the $I^{\text {th }}$ fracture (resp $J^{\text {th }}$ ) in two halves denoted arbitrary by the suffix $\ell$ and $r$ (for left and right). We introduce also the normal $\mathbf{n}_{\cap_{I J} I \ell}$ as being the normal to intersection $\cap_{I J}$ pertaining to the plane of the $I^{\text {th }}$ fracture, pointing in the $\ell$ direction. In addition, it is possible to introduce the $\nabla_{I}$ gradient operator as being the $2 D$ gradient operator operating only in the $I^{\text {th }}$ fracture plane. When there is no ambiguity, we will remove the index $I$ to this operator.

We denote the considered limit as $\lim _{\varepsilon \rightarrow 0}\left(p_{\varepsilon}(\mathbf{r})\right)=p(\mathbf{r})$. We argue that $p(\mathbf{r})$ is the solution of the following problem:

$$
\forall I=1, \cdots, N, \quad \nabla_{I} \cdot\left(k_{I} \nabla_{I} p(\mathbf{r})\right)=g_{I}(\mathbf{r})
$$

The notation $\nabla_{I} \ldots$ corresponds to the 2D gradient operator defined in the fracture. In order to get a meaningful limit, we must specify boundary conditions at the frontiers of the computational domain, at the boundary of each fracture, and finally at the intersections between fractures.

The boundary condition at the frontier $\Omega$ of the computational domain remains essentially unchanged (notice that the same reasoning should hold also when using mixed Dirichlet Neumann conditions). Considering now each fracture, the boundary value problem to be solved is $2 D$. The position vector $\mathbf{r}$ is essentially $2 D$. In particular, the boundary condition at the border of each fracture, say $\partial \Omega_{I}$ in the $I^{\text {th }}$ fracture plane, (a $1 D$ curve, corresponding for example 
to an ellipse in the case of elliptic fractures) can be written as:

$$
k_{I} \nabla p(\mathbf{r}) \cdot \mathbf{n}=0,
$$

where $\mathbf{n}$ is the outward normal to the boundary (FIG. 6). Notice that in the present formulation, the two initial faces of the fracture in direct contact with the matrix do not play any role.

In order to get a well-defined problem, a boundary condition must be specified at every intersection between fractures. In [1], the following condition was proposed:

$$
\mathbf{n}_{\cap_{I J} I \ell} \cdot k_{I}\left[\nabla_{I} p\left(\mathbf{r}_{\cap_{I J}}(x)\right)_{\ell}-\nabla_{I} p\left(\mathbf{r}_{\cap_{I J}}(x)\right)_{r}\right]+\mathbf{n}_{\cap_{I J} J \ell} \cdot k_{J}\left[\nabla_{J} p\left(\mathbf{r}_{\cap_{I J}}(x)\right)_{\ell}-\nabla_{J} p\left(\mathbf{r}_{\cap_{I J}}(x)\right)_{r}\right]=0 .
$$

It means physically that at each location of the intersection, the four fluxes converging at the considered position must balance. The subscript $\ell$ and ${ }_{r}$ (left and right) account for the two sides of the intersection. The normal $\mathbf{n}_{\cap_{I J} I}$ is one normal vector to the intersection under consideration lying in the plane of the I th fracture involved in the intersection. This condition reflects that due to the small transverse area of the intersection, longitudinal flow in the intersection will become negligible as $\varepsilon \rightarrow 0$, independently on the value of permeability value at the intersection. Note that the same argument can be followed in the case of a transient problem, because the volume integral of the accumulation term inside the intersection will also become negligible.

\section{The projection method.}

We are in position to build an approximation scheme allowing us to eliminate internal degrees of freedom inside each fracture. In a finite element solution framework, using an explicit mesh of each fracture, these degrees of freedom will correspond to the unknowns associated with the generic element inside each fracture. The basic idea is to express these lumped degrees of freedom as a function of the pressure trace at intersections. Thus, using the boundary condition (A2), we get equations coupling only degrees of freedom attached to intersections. In order to proceed, we focus our attention over the $I^{\text {th }}$ fracture called $\Omega_{I}$ of the set. In order to simplify the analysis, we suppose that this fracture intersects $n_{\cap I}$ other fractures by simple intersections restricted to be segments. So, the $n_{\cap I}$ intersections are non-intersecting segments denoted by $\cap_{j}$ of arbitrary lengths, the label of which belong to a subset of the $N_{\cap}$ labels denoted $J_{I}$, such that $\operatorname{Card} J_{I}=n_{\cap I}$. For the intersection labeled by $i$, we call $I(i)$ the label of the other fracture 
that is intercepted by $I$ (FIG. 6). So we have $J(i)=J_{I} \cup J_{I}(i)$. By hypothesis, this fracture is well defined.

For each intersection segment, we introduce a complete set of basis functions, denoted by $\Phi_{j}^{n}(x)$. Here, the integer $n=1, \cdots \infty$ labels the function, while $j=1, \cdots, n_{\cap I}$ labels the intersection number. We add two conditions:

$$
\begin{aligned}
\Phi_{j}^{1}(x) & =1, \\
\int_{\cap_{j}} \Phi_{j}^{n}(x) d x & =0 \quad \text { if } \quad n \geqslant 2 .
\end{aligned}
$$

Possible choices could involve sine and cosine functions, or polynomial families like Legendre or Tchebychev. Notice that up to a dilatation due to the varying length of the intersection, the same set of functions can be retained for every intersection between any fractures. We introduce elementary solutions defined by $\hat{P}_{j}^{n}(\mathrm{r})$, solution of the following boundary value problem:

$$
\left\{\begin{array}{l}
\nabla_{I} \cdot\left(k_{I} \nabla \hat{P}_{j}^{n}(\mathbf{r})\right)=0, \\
k_{I} \nabla \hat{P}_{j}^{n}(\mathbf{r}) \cdot \mathbf{n}=\mathbf{0} \quad \text { on } \quad \partial \Omega_{I}, \\
\hat{P}_{j}^{n}(\mathbf{r})=\delta_{i j} \times \Phi_{j}^{n}(\mathbf{r}) \quad \text { if } \quad \mathbf{r} \in \cap_{i}, \quad \forall i=1, n_{\cap} .
\end{array}\right.
$$

Here, $\delta_{i j}$ is the Kronecker symbol. These functions are perfectly defined, as being the unique solution of a Dirichlet Neumann problem. We can decompose the pressure along the $j^{\text {th }}$ intersection:

$$
p_{\cap_{j}}(x)=\sum_{n=1}^{\infty} P_{j}^{n} \Phi_{j}^{n}(x)
$$

and we define $p_{\cap}(\mathbf{r})$ as the solution of the Laplace equation, without source term but with the imposed profiles at the intersections. We have:

$$
p_{\cap}(\mathbf{r})=\sum_{j \in J_{I}} \sum_{n=1}^{\infty} P_{j}^{n} \hat{P}_{j}^{n}(\mathbf{r}) .
$$

375 We consider now the complete Laplace limit problem (A1). We suppose that the pressures profiles at all the existing intersections are known: $P_{j}(\mathbf{r})$. Using the linearity of the Laplace equation, and the boundary conditions, we showed 
that:

$$
p(\mathbf{r})=\sum_{j \in J_{I}} \sum_{n=1}^{\infty} P_{j}^{n} \hat{P}_{j}^{n}(\mathbf{r})+\int_{\Omega_{I}} d^{2} \mathbf{r}^{\prime} B\left(\mathbf{r}, \mathbf{r}^{\prime}\right) \times g_{I}\left(\mathbf{r}^{\prime}\right)
$$

Here the Green's function $B\left(\mathbf{r}, \mathbf{r}^{\prime}\right)$ is an elementary solution of the Laplace problem, with a source term $\delta\left(\mathbf{r}-\mathbf{r}^{\prime}\right)$ :

$$
\forall I=1, \cdots, N, \quad \nabla_{\mathbf{r}} \cdot\left(k_{I} \nabla_{\mathbf{r}} B\left(\mathbf{r}, \mathbf{r}^{\prime}\right)\right)=\delta\left(\mathbf{r}-\mathbf{r}^{\prime}\right)
$$

to be solved with the following boundary conditions:

$$
\begin{aligned}
k_{I} \nabla_{\mathbf{r}} B\left(\mathbf{r}, \mathbf{r}^{\prime}\right) \cdot \mathbf{n} & =0 \quad \text { for } \quad \mathbf{r} \in \partial \Omega_{I}, \\
B\left(\mathbf{r}, \mathbf{r}^{\prime}\right) & =0 \quad \text { if } \quad \mathbf{r} \in \cap_{i}, \quad \forall i=1, n_{\cap} .
\end{aligned}
$$

380 One must remember that it is a $2 D$ Green's function, because the working space is the space of the fracture. This is 381 the general form of the solution with source term, but we still need relations to determine the set of $P_{j}^{n}$ values. This 382 will be done by using the boundary condition (A2) in next subsection.

383 In order to get equations allowing to determine the unknowns $P_{i}^{m}$, we use the boundary condition (A2), conveniently 384 projected on the basis function $\Phi_{i}^{m}(x)$. The projection gives the following relation: $\forall i=1, \cdots, n_{\cap}, \quad \forall m=1, \cdots, \infty$,

$$
\begin{aligned}
\int_{\cap_{i}} d x \mathbf{n}_{\cap_{I J} I \ell} \cdot & \left.k_{I}(\mathbf{r}(x))\left[\nabla_{I} p\left(\mathbf{r}_{\cap_{I J}}(x)\right)_{\ell}-\nabla_{I} p\left(\mathbf{r}_{\cap_{I J}}(x)\right)_{r}\right)\right] \times \Phi_{i}^{m}(x) \\
& +\int_{\cap_{i}} d x \mathbf{n}_{\cap_{I J} J} \cdot k_{J}(\mathbf{r}(x))\left[\nabla_{J} p\left(\mathbf{r}_{\cap_{I J}}(x)\right)_{\ell}-\nabla_{J} p\left(\mathbf{r}_{\cap_{I J}}(x)\right)_{r}\right] \times \Phi_{i}^{m}(x)=0 .
\end{aligned}
$$

${ }_{385}$ This allows us to get an infinite set of relations, by inserting (A8) in the projection of the boundary conditions (A10) $386: \forall i, \cdots, m$

$$
\sum_{j \in J(i)} \sum_{n=1}^{\infty} T_{i j}^{m n} \times P_{j}^{n}+\int_{\Omega_{I}} d^{2} \mathbf{r}^{\prime} B_{i}^{m}\left(\mathbf{r}^{\prime}\right) \times g_{I}\left(\mathbf{r}^{\prime}\right)+\int_{\Omega_{I(i)}} d^{2} \mathbf{r}^{\prime} B_{i}^{m}\left(\mathbf{r}^{\prime}\right) \times g_{I(i)}\left(\mathbf{r}^{\prime}\right)=0
$$

${ }_{387}$ or, equivalently, introducing $B_{i}^{m}=-\int_{\Omega_{I}} d^{2} \mathbf{r}^{\prime} B_{i}^{m}\left(\mathbf{r}^{\prime}\right) \times g_{I}\left(\mathbf{r}^{\prime}\right)-\int_{\Omega_{I(i)}} d^{2} \mathbf{r}^{\prime} B_{i}^{m}\left(\mathbf{r}^{\prime}\right) \times g_{I(i)}\left(\mathbf{r}^{\prime}\right):$ 


$$
\sum_{j \in J(i)} \sum_{n=1}^{\infty} T_{i j}^{m n} \times P_{j}^{n}=B_{i}^{m}
$$

In order to emphasize the overall linearity of the problem, we have introduced the following quantities $T_{i j}^{n m}$ and $B_{i}^{m}$ as:

$$
\begin{aligned}
T_{i j}^{m n} & =\int_{\cap_{i}} d x \mathbf{n}_{\cap_{I J} I \ell} \cdot k_{I}(\mathbf{r}(x))\left[\nabla_{I} \hat{P}_{j}^{n}(\mathbf{r}(x))_{\ell}-\nabla_{I} \hat{P}_{j}^{n}(\mathbf{r}(x))_{r}\right] \times \Phi_{i}^{m}(x), \\
B_{i}^{m}\left(\mathbf{r}^{\prime}\right) & =\int_{\cap_{i}} d x \mathbf{n}_{\cap_{I J} I \ell} \cdot k_{I}(\mathbf{r}(x))\left[\nabla_{I} B\left(\mathbf{r}(x), \mathbf{r}^{\prime}\right)_{\ell}-\nabla_{I} B\left(\mathbf{r}(x), \mathbf{r}^{\prime}\right)_{r}\right] \times \Phi_{i}^{m}(x) .
\end{aligned}
$$

It was shown in [1], and the proof is presented in the appendix $\mathrm{C}$ that $T_{i j}^{n m}$ and $B_{i}^{m}\left(\mathbf{r}^{\prime}\right)$ may be written under a much more simple and explicit form:

$$
\begin{aligned}
T_{i j}^{m n} & =\int_{\Omega_{I}} d^{2} \mathbf{r} k_{I}(\mathbf{r}) \nabla_{I} \hat{P}_{i}^{m}(\mathbf{r}) \cdot \nabla_{I} \hat{P}_{j}^{n}(\mathbf{r}), \\
B_{i}^{m}\left(\mathbf{r}^{\prime}\right) & =\hat{P}_{i}^{m}\left(\mathbf{r}^{\prime}\right) .
\end{aligned}
$$

The notation $\sum^{\prime}$ means that the summation must be performed over both fractures involved by the $i^{\text {th }}$ intersection of the $I^{t h}$ fracture. Here, the notation $I(i)$ denotes that we are considering the moment of the solution over the $i^{\text {th }}$ intersection, between the $I^{t h}$ fracture, and the $I(i)^{t h}$ intersection. It is this summation over all the involved fractures that permits to ensure mass conservation at the intersections. In order to simplify the presentation, we did not reintroduce the labels of $\mathrm{I}$ and $\mathrm{J}$ th fractures. In practice, one will truncate the order $n$ of the approximation by restricting $m, n \leqq n_{0}$. At the end of the process, we will have to solve a linear system of $N_{\cap} \times n_{0}$ equations. To the lowest order approximation $n_{0}=1$, will correspond $N_{\cap}$ equations to be solved. This corresponds well to our initial program, this approximation will be studied in more details in Section 7.

The algebraic form (A15) permits to check by direct inspection that we have the general symmetry relation:

$$
T_{i j}^{m n}=T_{j i}^{n m}
$$

for all labels $i, j, m$ and $n$.

It can be checked by inspection of this formula that the matrix $T_{i j}^{11}$ is symmetric, positive. It is not definite because 
we have the general relation:

$$
\sum_{j} T_{i j}^{11}=0
$$

This equality can be derived by noticing that we have the general sum rule:

$$
P(\mathbf{r})=\sum_{j=1, n_{\cap I}} \hat{P}_{j}^{1}(\mathbf{r})=1
$$

We come back to the full transient diffusion problem 3 on the fracture network. Our present goal is to build an approximation scheme valid for time scales greater to a typical diffusion time over one fracture. For a typical fracture $I$ of permeability $k_{I}$ and of size $L_{I}$, this time scale is of order $L_{I}^{2} / D_{I}$, here the diffusion coefficient is given by $D_{I}=k_{I} / \varphi \mu c_{t}$. Let $p(\mathbf{r}, t)$ be the solution of the full diffusion problem. We write $p(\mathbf{r}, t)$ under the following form:

$$
p(\mathbf{r}, t)=\delta p(\mathbf{r}, t)+\sum_{j \in J_{I}} \sum_{n=1}^{\infty} P_{j}^{n}(t) \hat{P}_{j}^{n}(\mathbf{r})+\int_{\Omega_{I}} d^{2} \mathbf{r}^{\prime} B\left(\mathbf{r}, \mathbf{r}^{\prime}\right) \times g_{I}\left(\mathbf{r}^{\prime}\right) .
$$

with an additional condition:

$$
\delta p(\mathbf{r}, t)=0 \quad \text { if } \quad \mathbf{r} \in \cup \cap_{i} .
$$

The definition of $\hat{P}_{j}^{n}(\mathbf{r})$ explains the overall simplification. Now, we can use the pseudo steady state assumption ${ }_{416}$ to drop the partial time derivative in the LHS. It means physically that the pressure inside a given fracture follows a 
${ }_{417}$ steady state problem with a source term given by the term $\left(\varphi \mu c_{t} \sum_{j}^{n_{\cap I}} \sum_{n=1}^{\infty} \dot{P}_{j}^{n}(t) \hat{P}_{j}^{n}(\mathbf{r})\right)$. We get:

$$
\nabla \cdot\left(k_{I} \nabla \delta p(\mathbf{r}, t)\right)=\varphi \mu c_{t} \sum_{j \in J_{I}} \sum_{n=1}^{\infty} \dot{P}_{j}^{n}(t) \hat{P}_{j}^{n}(\mathbf{r})
$$

418 In this expression, the term $\left(\varphi \mu c_{t} \sum_{j \in J_{I}} \sum_{n=1}^{\infty} \dot{P}_{j}^{n}(t) \hat{P}_{j}^{n}(\mathbf{r})\right)$ is a surface source term that appears due to the changing forcing term at the intersections. It specifies the form of the $g_{I}(\mathbf{r})$. Using the general solution with source term (A8), 420 we get thus the following solution:

$$
p(\mathbf{r}, t)=\sum_{j \in J_{I}} \sum_{n=1}^{\infty} P_{j}^{n}(t) \hat{P}_{j}^{n}(\mathbf{r})+\int_{\Omega_{I}} d^{2} \mathbf{r}^{\prime} B\left(\mathbf{r}, \mathbf{r}^{\prime}\right) \times g_{I}\left(\mathbf{r}^{\prime}\right)+\int_{\Omega_{I}} d^{2} \mathbf{r}^{\prime} B\left(\mathbf{r}, \mathbf{r}^{\prime}\right) \times \sum_{j \in J_{I}} \sum_{n=1}^{\infty} \varphi \mu c_{t} \dot{P}_{j}^{n}(t) \hat{P}_{j}^{n}\left(\mathbf{r}^{\prime}\right)
$$

$$
\begin{aligned}
\forall i, m, \sum_{j \in J(i)} \sum_{n=1}^{\infty} T_{i j}^{m n} \times & P_{j}^{n}+\int_{\Omega_{I}} d^{2} \mathbf{r} B_{i}^{m}(\mathbf{r}) \times g_{I}\left(\mathbf{r}^{\prime}\right)+\int_{\Omega_{I}} d^{2} \mathbf{r} \dot{B}_{i}^{m}(\mathbf{r}) \times \sum_{j \in J(i)} \sum_{n=1}^{\infty} \varphi \mu c_{t} \dot{P}_{j}^{n}(t) \hat{P}_{j}^{n}(\mathbf{r}) \\
& +\int_{\Omega_{I(i)}} d^{2} \mathbf{r} B_{i}^{m}(\mathbf{r}) \times g_{I}\left(\mathbf{r}^{\prime}\right)+\int_{\Omega_{I(i)}} d^{2} \mathbf{r} B_{i}^{m}(\mathbf{r}) \times \sum_{j \in J(i)} \sum_{n=1}^{\infty} \varphi \mu c_{t} \dot{P}_{j}^{n}(t) \hat{P}_{j}^{n}(\mathbf{r})=0 .
\end{aligned}
$$

${ }_{424}$ Let us introduce the "mass matrix" $K_{i j}^{m n}$ by means of the definition:

$$
K_{i j}^{m n}=\int_{\Omega_{I}} d^{2} \mathbf{r} B_{i}^{m}(\mathbf{r}) \times \varphi \mu c_{t} \hat{P}_{j}^{n}(\mathbf{r})=\varphi \mu c_{t} \int_{\Omega_{I}} d^{2} \mathbf{r} \hat{P}_{i}^{m}(\mathbf{r}) \hat{P}_{j}^{n}(\mathbf{r})
$$

${ }_{425}$ Here, we have used directly the equality (A16). The set of equations can be rewritten under a more synthetic form:

$$
\forall i, m, \quad \sum_{j \in J(i)} \sum_{n=1}^{\infty} K_{i j}^{m n} \times \frac{d P_{j}^{n}(t)}{d t}=\sum_{j \in J(i)} \sum_{n=1}^{\infty} T_{i j}^{m n} \times P_{j}^{n}+B_{i}^{m} .
$$


${ }_{427}$ the base solutions:

$$
\begin{aligned}
T_{i j}^{m n} & =\int_{\Omega_{I}} d^{2} \mathbf{r} k_{I}(\mathbf{r}) \nabla_{I} \hat{P}_{i}^{m}(\mathbf{r}) \cdot \nabla_{I} \hat{P}_{j}^{n}(\mathbf{r}), \\
K_{i j}^{m n} & =\varphi \mu c_{t} \int_{\Omega_{I}} d^{2} \mathbf{r} \hat{P}_{i}^{m}(\mathbf{r}) \hat{P}_{j}^{n}(\mathbf{r}) .
\end{aligned}
$$

${ }_{428} T_{i j}^{m n}$ and $K_{i j}^{m n}$ appear as "scalar products" of the basic solutions or of their gradients. These expressions appear also ${ }_{429}$ in a finite element context [43]. The symmetry and positiveness of the matrices $\mathbf{K}$ and $\mathbf{T}$ can be checked by direct 430 inspection:

$$
\begin{aligned}
T_{i j}^{m n} & =T_{j i}^{n m}, \\
K_{i j}^{m n} & =K_{j i}^{n m},
\end{aligned}
$$

${ }_{431}$ for all labels $i, j, m$ and $n$. Direct numerical methods can be set up to determine the transmissitivity $T_{i j}^{m n}$ and the 432 mass matrix $K_{i j}^{m n}$. Each Laplace boundary value problem (A5) can be solved on each fracture independently of the ${ }_{433}$ others once the intersection segments have been determined. This means that internal degrees of freedom between ${ }_{434}$ different fractures are not directly coupled. This is quite natural, as all the information must be carried by the ${ }_{435}$ intersections. The boundary value problem (A5) can be solved using for example a finite element code by meshing ${ }_{436}$ only the $I^{\text {th }}$ fracture, once for all $i, j, m$ and $n$, plus a Laplace equation solver. High values of $m$ and $n$ will probably ${ }_{437}$ need highly refined meshes, corresponding to having a high level of details. The same procedure must evidently 438 be repeated for every fracture, leading to a numerical cost proportional to twice the total number of intersections.

439 Finding a method to control the accuracy of the method as a function of $m$ and $n$ would be of theoretical interest. ${ }_{440}$ Fast evaluations methods of $\mathbf{K}$ and $\mathbf{T}$ remain an open area of work followed by Khvoenkova and Delorme [42]. The ${ }_{441}$ similar approach followed in $2 D$, with fracture intersections that degenerate as single points is exact, and corresponds ${ }_{442}$ to a so called resistor/network model developed by [31]. 
We want to show that:

$$
\begin{aligned}
T_{i j}^{m n} & =\int_{\Omega_{I}} d^{2} \mathbf{r} k_{I}(\mathbf{r}) \nabla_{I} \hat{P}_{i}^{m}(\mathbf{r}) \cdot \nabla_{I} \hat{P}_{j}^{n}(\mathbf{r}), \\
B_{i}^{m}\left(\mathbf{r}^{\prime}\right) & =\hat{P}_{i}^{m}\left(\mathbf{r}^{\prime}\right) .
\end{aligned}
$$

We start from the definitions:

$$
\begin{aligned}
T_{i j}^{m n} & =\int_{\cap_{i}} d x \mathbf{n}_{\cap_{I J} I \ell} \cdot k_{I}(\mathbf{r}(x))\left[\nabla_{I} \hat{P}_{j}^{n}(\mathbf{r}(x))_{\ell}-\nabla_{I} \hat{P}_{j}^{n}(\mathbf{r}(x))_{r}\right] \times \Phi_{i}^{m}(x), \\
B_{i}^{m}\left(\mathbf{r}^{\prime}\right) & =\int_{\cap_{i}} d x \mathbf{n}_{\cap_{I J} I \ell} \cdot k_{I}(\mathbf{r}(\mathbf{x}))\left[\nabla_{I} B\left(\mathbf{r}(x), \mathbf{r}^{\prime}\right)_{\ell}-\nabla_{I} B\left(\mathbf{r}(x), \mathbf{r}^{\prime}\right)_{r}\right] \times \Phi_{i}^{m}(x),
\end{aligned}
$$

with $P_{i}^{m}(\mathbf{r})$ which is solution of the following boundary value problem:

$$
\left\{\begin{array}{l}
\nabla_{I} \cdot\left(k_{I} \nabla \hat{P}_{i}^{m}(\mathbf{r})\right)=0, \\
k_{I} \nabla \hat{P}_{i}^{m}(\mathbf{r}) \cdot \mathbf{n}=0 \quad \text { on } \quad \partial \Omega_{I}, \\
\hat{P}_{i}^{m}(\mathbf{r})=\delta_{i j} \times \Phi_{i}^{m}(\mathbf{r}) \quad \text { if } \quad \mathrm{r} \in \cap_{i}, \quad \forall j=1, n_{\cap} .
\end{array}\right.
$$

${ }_{447}$ These equalities may be derived by remarking that $T_{i j}^{m n}$ and $B_{i}^{m}\left(\mathbf{r}^{\prime}\right)$ can be rewritten under a slightly different form:

$$
\begin{aligned}
T_{i j}^{m n} & =\int_{\cap_{i}} d x \mathbf{n} \cdot k_{I}(\mathbf{r}(x))\left[\nabla_{I} \hat{P}_{j}^{n}(\mathbf{r}(x))-\nabla_{I} \hat{P}_{j}^{n}(\mathbf{r}(x))_{r}\right] \times \Phi_{i}^{m}(x) \\
& =\int_{\cap_{i}} d x \mathbf{n} \cdot k_{I}(\mathbf{r}(x)) \nabla_{I} \hat{P}_{j}^{n}(\mathbf{r}(x)) \times \hat{P}_{i}^{m}(\mathbf{r}(x)) \\
& =\int_{\bigcup \cap_{k} \cup \partial \Omega_{I}} d r \mathbf{n} \cdot k_{I}(\mathbf{r}(x)) \nabla_{I} \hat{P}_{j}^{n}(\mathbf{r}(x)) \times \hat{P}_{i}^{m}(\mathbf{r}(x)) \\
B_{i}^{m}\left(\mathbf{r}^{\prime}\right) & =\int_{\bigcup \cap_{k} \cup \partial \Omega_{I}} d x \mathbf{n} \cdot k_{I}(\mathbf{r}(x)) \nabla_{I} B\left(\mathbf{r}(x), \mathbf{r}^{\prime}\right) \times \hat{P}_{i}^{m}(\mathbf{r}(x)) .
\end{aligned}
$$

In order to understand the second and fourth equalities, the reader must imagine that the intersection contour is a closed 2D contour of small thickness allowing to replace the contribution of left and right fluxes on the two sides of the intersection by a contour integral that allows to use Green's theorem. We did not change the notations in order to simplify the presentation. In present form, the boundary integrals are extended on all the frontiers associated with the $I^{\text {th }}$ fracture, so we can use Green's theorem in order to transform the contour integral on a surface integral over 
453 the fracture domain. We obtain:

$$
T_{i j}^{m n}=\int_{\Omega_{I}} d^{2} \mathbf{r} \nabla \cdot\left[k_{I}(\mathbf{r}) \nabla_{I} \hat{P}_{j}^{n}(\mathbf{r}) \times \hat{P}_{i}^{m}(\mathbf{r})\right]
$$

${ }_{454}$ from which the desired identity follows, using once again the local equation obeyed by $\hat{P}_{j}^{n}(\mathbf{r})$ :

$$
T_{i j}^{m n}=\int_{\Omega_{I}} d^{2} \mathbf{r} k_{I}(\mathbf{r}) \nabla_{I} \hat{P}_{j}^{n}(\mathbf{r}) \cdot \nabla \hat{P}_{i}^{m}(\mathbf{r})
$$

${ }_{455}$ For $B_{i}^{m}\left(\mathbf{r}^{\prime}\right)$ we need some additional manipulations in the same style:

$$
\begin{aligned}
B_{i}^{m}\left(\mathbf{r}^{\prime}\right) & =\int_{\bigcup \cap_{k} \cup \partial \Omega_{I}} d x \mathbf{n} \cdot k_{I}(\mathbf{r}) \nabla_{I} B\left(\mathbf{r}(x), \mathbf{r}^{\prime}\right) \times \hat{P}_{i}^{m}(\mathbf{r}(x)) \\
& =\int_{\Omega_{I}} d^{2} \mathbf{r} \nabla \cdot\left[k_{I}(\mathbf{r}) \nabla_{I} B\left(\mathbf{r}, \mathbf{r}^{\prime}\right) \times \hat{P}_{i}^{m}(\mathbf{r})\right] .
\end{aligned}
$$

${ }_{456}$ Using the equation defining $B\left(\mathbf{r}, \mathbf{r}^{\prime}\right)$, we obtain:

$$
B_{i}^{m}\left(\mathbf{r}^{\prime}\right)=\hat{P}_{i}^{m}\left(\mathbf{r}^{\prime}\right)+\int_{\Omega_{I}} d^{2} \mathbf{r} k_{I}(\mathbf{r}) \nabla_{I} B\left(\mathbf{r}, \mathbf{r}^{\prime}\right) \cdot \nabla \hat{P}_{i}^{m}(\mathbf{r})
$$

457 The second term of the RHS may be written under a more explicit form:

$$
\begin{aligned}
\int_{\Omega_{I}} d^{2} \mathbf{r} k_{I}(\mathbf{r}) \nabla_{I} B\left(\mathbf{r}, \mathbf{r}^{\prime}\right) \cdot \nabla \hat{P}_{i}^{m}(\mathbf{r})= & \int_{\Omega_{I}} d^{2} \mathbf{r} k_{I}(\mathbf{r}) \nabla_{I} B\left(\mathbf{r}, \mathbf{r}^{\prime}\right) \cdot \nabla \hat{P}_{i}^{m}(\mathbf{r}) \\
& +\int_{\Omega_{I}} d^{2} \mathbf{r} B\left(\mathbf{r}, \mathbf{r}^{\prime}\right) \nabla_{I} \cdot\left[k_{I}(\mathbf{r}) \nabla \hat{P}_{i}^{m}(\mathbf{r})\right] \\
= & \int_{\Omega_{I}} d^{2} \mathbf{r} \nabla_{I} \cdot\left[k_{I}(\mathbf{r}) B\left(\mathbf{r}, \mathbf{r}^{\prime}\right) \nabla \hat{P}_{i}^{m}(\mathbf{r})\right] \\
= & \int_{\bigcup \cap_{k} \cup \partial \Omega_{I}} d r k_{I}(\mathbf{r}) B\left(\mathbf{r}, \mathbf{r}^{\prime}\right) \times \nabla_{I} \hat{P}_{i}^{m}(\mathbf{r}) \cdot \mathbf{n}
\end{aligned}
$$

${ }_{458}$ But the last expression is equal to zero, thanks to the boundary conditions on $B\left(\mathbf{r}, \mathbf{r}^{\prime}\right)$ and $k_{I}(\mathbf{r}) \nabla_{I} \hat{P} P_{i}^{m}(\mathbf{r}) \cdot \mathbf{n}$ on ${ }_{459} \bigcup \cap_{k} \bigcup \partial \Omega_{I}$

$$
\int_{\cup \cap_{k} \cup a \Omega_{I}} d r k_{I}(\mathbf{r}) B\left(\mathbf{r}, \mathbf{r}^{\prime}\right) \times \nabla_{I} \hat{P}_{i}^{m}(\mathbf{r}) \cdot \mathbf{n}=0
$$

${ }_{460}$ This provides the announced result. 
[1] B. Noetinger, N. Jarrige A quasi steady state method for solving transient Darcy flow in complex 3D fractured networks Journal of Computational Physics, Volume 231, Issue 1, 1 January 2012, Pages 23-38, ISSN 0021-9991, $10.1016 / \mathrm{j} . \mathrm{jcp} .2011 .08 .015$.

[2] G.I. Barenblatt, I.P. Zheltov, and I.N. Kochina. Basic concepts in the theory of seepage of homogeneous liquids in fissured rocks. Journal of Applied Mathematics and Mechanics, 24(5):1286-1303, 1960

[3] J.C. Sabathier, B.J. Bourbiaux, MC Cacas, and S. Sarda. A new approach of fractured reservoirs. SPE Paper $\sharp 39825$ International Petroleum Conference and Exhibition of Mexico, 1998.

[4] B.J. Bourbiaux, S. Granet, P. Landereau, B. Noetinger, S. Sarda, J.C. Sabathier, Scaling Up Matrix-Fracture Transfers in Dual-Porosity Models: Theory and Application SPE Paper $\sharp 56557$ SPE Annual Technical Conference and Exhibition, 3-6 October 1999, Houston, Texas

(5) P.M. Adler and J.F. Thovert. Fractures and Fracture Networks. Kluwer, Dordrecht, 1999.

[6] S. Sarda, L. Jeannin, R. Basquet, and B.J. Bourbiaux. Hydraulic characterization of fractured reservoirs: Simulation on discrete fracture models. SPE Paper $\sharp 73300$, Reservoir Evaluation \& Engineering, Vol 5(2) 154-162, April 2002.

[7] S.P. Neuman. Trends, prospects and challenges in quantifying flow and transport through fractured rocks. Hydrogeology Journal, 13(1):124-147, 2005.

[8] B.J. Bourbiaux Fractured Reservoir Simulation: a Challenging and Rewarding Issue Oil Gas Sci. Technol. - Rev. IFP 65 2 (2010) 227-238 DOI: $10.2516 /$ ogst/2009063

[9] P. Lemonnier, B. Bourbiaux Simulation of Naturally Fractured Reservoirs. State of the Art - Part 1 - Physical Mechanisms and Simulator Formulation. Oil Gas Sci. Technol.- Rev. IFP 652 (2010) 239-262 DOI: 10.2516/ogst/2009066

[10] P. Lemonnier, B. Bourbiaux Simulation of Naturally Fractured Reservoirs. State of the Art - Part 2 - Matrix-Fracture Transfers and Typical Features of Numerical Studies Oil Gas Sci. Technol. - Rev. IFP 652 (2010) 263-286 DOI: $10.2516 /$ ogst $/ 2009067$

[11] T. Arbogast, J. Douglas Jr, and U. Hornung. Derivation of the double-porosity model of single-phase flow via homogenization theory. SIAM J. Math Anal., 21(4):823-836, July 1990.

[12] M. Panfilov. Averaged model-type transition in flows through multiple heterogeneous porous media. C.R. Acad. Sci. Paris, 318(II):1437-1443, 1994.

[13] P. Royer, J.L. Auriault, and C. Boutin. Macroscopic modeling of double-porosity reservoirs. Journal of Petroleum Science and Engineering, 16(4):187-202, 1996.

[14] R.E. Showalter. In Homogenization and Porous Media, volume 6 of Interdisciplinary Applied Mathematics Series, chapter Micro-structure Models of Porous Media. Springer, New York, 1997.

[15] M. Quintard and S. Whitaker. Transport in chemically and mechanically heterogeneous porous media I: Theoretical development of region-averaged equations for slightly compressible single-phase flow. Advances in Water Resources, $19(1): 29-47,1996$.

[16] M. Quintard and S. Whitaker. Transport in chemically and mechanically heterogeneous porous media ii: Comparison with numerical experiments for slightly compressible single-phase flow. Advances in Water Resources, 19(1):49-60, 1996.

[17] P. Landereau, B. Noetinger, and M. Quintard. Quasi-steady two-equation models for diffusive transport in fractured porous media: large-scale properties for densely fractured systems. Advances in Water Resources, 24 (8):863-876, 2001.

[18] J.F. McCarthy. Effective permeability of sandstone-shale reservoirs by a random walk method. J. Phys. A: Math. Gen., 23:445-451, 1990 .

[19] J.F. McCarthy. Continuous-time random walks on random media. J. Phys. A: Math. Gen., 26:2495-2503, 1993.

[20] B. Noetinger and T. Estebenet. Up scaling of fractured porous media using a continuous time random walk method. Transport in Porous Media, 39:315-337, 2000.

[21] B. Noetinger, T. Estebenet, and M. Quintard. Up scaling of fractured media: Equivalence between the large scale averaging theory and the continuous time random walk method. Transport in Porous Media, 43:581-596, 2001.

[22] B. Noetinger, T. Estebenet, and P. Landereau. A direct determination of the transient exchange term of fractured media using a continuous time random walk method. Transport in Porous Media, 44:539-557, 2001.

[23] A de Swaan Analytic solutions for determining naturally fractured reservoir properties by well testing SPE Journal, 54 117-22 1976

[24] A de Swaan Influence of shape and skin of matrix-rock blocks on pressure transients in fractured reservoirs SPE formation evaluation, 54 344-352 1990

[25] A De Swann. and Ramirez-Villa, M. Functions of flow from porous rock blocks. Journal of Petroleum Science and Engineering, vol. 9, no 1, p. 39-48. 1993

[26] F.Daviau Interprétation des essais de puits : les méthodes nouvelles ed Technip, Paris 1986

[27] L. De Arcangelis, J. Koplik, S. Redner, and D. Wilkinson. Hydrodynamic Dispersion in Network Models of Porous Media. Phys. Rev. Lett., 57:986-999, 1986.

[28] J. Koplik, S. Redner, and D. Wilkinson. Transport and dispersion in random networks with percolation disorder. Physical Review A, 37:2619-2636, 1988.

[29] B. Berkowitz and I. Balberg. Percolation theory and its application to groundwater hydrology. Water Res. Res., 29(4):775794, 1993.

[30] J. Chang and Y.C. Yortsos Pressure transient analysis of fractal reservoirs. SPE Paper $\sharp 18170$, SPE Form. Eval.,, Vol 5(1)pages 31-38, 1990. 
[31] J.A. Acuna and Y.C. Yortsos Application of fractal geometry to the study of networks of fractures and their pressure transient. Water Res. Res., 31 3:527-540, 1995.

[32] R.W. Zimmerman, G. Chen, T. Hadgu, and G.S. Bodvarsson. A numerical dual-porosity model with semi-analytical treatment of fracture/matrix flow. Water Res. Res., 29:2127-37, 1993.

[33] V.V. Mourzenko, J-F. Thovert, and P.M. Adler. Geometry of simulated fractures. Physical Review E, 53(6), 1996.

[34] J. Douglas, F. Pereira, and LM. Yeh. A parallelizable method for two-phase flows in naturally-fractured reservoirs. Comput. Geosci., 1(3-4):333-368, 1997.

[35] S. Granet, P. Fabrie, P. Lemonnier, and M. Quintard. A two-phase flow simulation of a fractured reservoir using a new fissure element method. Journal of Petroleum Science and Engineering, 32(1):35-52, 2001.

[36] A. Lange, R. Basquet, B. Bourbiaux, et al. Hydraulic characterization of faults and fractures using a dual medium discrete fracture network simulator. SPE Paper $\sharp 88675$ In 10th International Petroleum Exhibition and Conference, Abu Dhabi. SPE, 2002.

[37] T.N. Narasimhan and K. Pruess. MINC: An approach for analyzing transport in strongly heterogeneous systems. in Groundwater Flow and Quality Modeling. D. Reidel Publishing Co. Boston. 1988. p 375-391, 4 fig, 21 ref., 1988.

[38] I.I. Bogdanov, V.V. Mourzenko, J.F. Thovert, and P.M. Adler. Pressure drawdown well tests in fractured porous media. Water Res. Res., 39(1):1021, 2003a.

[39] A. Fourno, C. Grenier, F. Delay, E. Mouche, and H. Benabderrahmane. Smeared fractures: a promising approach to model transfers in fractured media. Developments in Water Science, 55:1003-1014, 2004.

[40] G. Pichot, J. Erhel and J. R. de Dreuzy, A mixed hybrid Mortar method for solving flow in discrete fracture networks, Applicable Analysis An International Journal, 89 Issue 10, 1629, doi:10.1080/00036811.2010.495333

[41] Y.S. Wu. Numerical simulation of single-phase and multiphase non-Darcy flow in porous and fractured reservoirs. Transport in porous media, 49(2):209-240, 2002.

[42] N. Khvoenkova and M. Delorme. An Optimal Method to Model Transient Flows in 3D Discrete Fracture Network. IAMG conference 2011 Salzburg, Austria, 2011.

[43] Y. Efendiev, J. Galvis, and X.H. Wu. Multiscale finite element methods for high-contrast problems using local spectral basis functions. J. Comput. Phys., 2302011 937-955.

[44] I.I. Bogdanov, V.V. Mourzenko, J.F. Thovert, and P.M. Adler. Effective permeability of fractured porous media in steady state flow. Water Resour. Res., 39(1):1023, 2003b.

[45] H. Stehfest. Algorithm 368: Numerical inversion of laplace transforms [d5]. Commun. ACM, 13:47-49, January 1970. 
